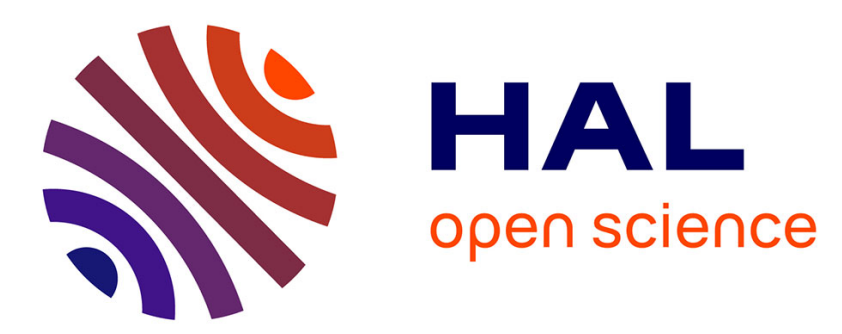

\title{
Robust transparent conducting electrode based on silver nanowire coating on polyelectrolytes multilayers
}

\author{
Jedsada Chavalitkul, Olivier Margeat, Jörg Ackermann, Stephan T Dubas
}

\section{To cite this version:}

Jedsada Chavalitkul, Olivier Margeat, Jörg Ackermann, Stephan T Dubas. Robust transparent conducting electrode based on silver nanowire coating on polyelectrolytes multilayers. Thin Solid Films, 2020, 711, pp.138272. 10.1016/j.tsf.2020.138272 . hal-02933272

\section{HAL Id: hal-02933272 \\ https://hal.science/hal-02933272}

Submitted on 8 Sep 2020

HAL is a multi-disciplinary open access archive for the deposit and dissemination of scientific research documents, whether they are published or not. The documents may come from teaching and research institutions in France or abroad, or from public or private research centers.
L'archive ouverte pluridisciplinaire HAL, est destinée au dépôt et à la diffusion de documents scientifiques de niveau recherche, publiés ou non, émanant des établissements d'enseignement et de recherche français ou étrangers, des laboratoires publics ou privés. 


\title{
Robust Transparent Conducting Electrode Based on Silver Nanowire Coating on Polyelectrolytes Multilayers
}

\author{
Jedsada Chavalitkul a , Olivier Margeat ${ }^{\text {b }}$, Jörg Ackermann ${ }^{\text {b }}$, Stephan T. Dubas ${ }^{\text {a,* }}$ \\ ${ }^{a}$ Petroleum and petrochemical college, Chulalongkorn university, Bangkok, 10330, Thailand \\ ${ }^{b}$ Aix Marseille Univ, CNRS, CINaM, Marseille, France \\ *Corresponding authors. \\ Email address: Stephan.d@,chula.ac.th (S.T. Dubas)
}

\begin{abstract}
Transparent conductive electrodes were fabricated by the deposition of silver nanowires (AgNW) on various substrates modified by the deposition of polyelectrolyte multilayers (PEM) as primer. The PEM primer films were coated on the glass substrate by the sequential deposition of 7 or 8 layers of poly(diallyl dimethyl ammonium chloride) (PDADMAC) and poly(styrene sulfonate) (PSS) to improve the adhesion of $\mathrm{AgNW}$ and produce flexible transparent conducting electrode. AgNW, with a diameter of 20-30 $\mathrm{nm}$ and 10-30 $\mu \mathrm{m}$ in length, were synthesized using a modified solvothermal method using glycerol and poly(vinyl pyrrolidone) (PVP) as reducing and capping agent. The physicochemical properties of the AgNW coated PEM were characterized using UV-Vis spectroscopy, atomic force microscope (AFM) and field emission scanning electron microscope (FESEM). The electrical conductivity of the layer was measured by 4 points probe and a standard scotch tape peeling test was used to investigate the stability of the coated AgNW on the PEM. The PEM 7 layers (with PDADMAC as outer layer) gave the best results to immobilize AgNW with the lowest sheet resistance (14 $\Omega /$ square) while
\end{abstract}


maintaining excellent transparency $(85 \%$ transmission at $550 \mathrm{~nm})$ even after up to 6 peeling test cycles. Finally, to demonstrate the benefit of this method, poly(ethylene terephthalate) (PET) sheet was coated with PEM primer and AgNW to produce flexible transparent conducting electrode.

Keywords: Transparent flexible electrode; Silver nanowires; Polyelectrolyte multilayers; Peeling test; Poly(ethylene terephthalate)

\section{Introduction}

Transparent conductive electrodes (TCE) are needed in electronic devices such as organic light emitting diodes (OLED) [1], solar cell [2-6], liquid crystal displays (LCD) [7, 8] as back electrode that allows light transmission either in or out of the cell. Typically, TCE are fabricated by the deposition of indium tin oxide (ITO) on a glass substrate which provides good transparency $(90 \%)$ in the visible range with an excellent conductivity (13 $\Omega /$ square $)[9,10]$. Because the ITO coatings are still expensive, alternatives solutions are being investigated. Several materials have been proposed to replace the ITO such as metal nanowires [11-14], carbon base electrode (carbon nanotube [15, 16], or graphene [17]), and hybrid material (ITO/silver nanowire [18], silver nanowire/metal mesh [19, 20] or silver nanowire/ZnO nanoparticle [21-23]). Silver nanoparticles and more precisely Ag nanowires (AgNW) are often described as one of the most promising alternatives due to excellent electrical conductivity and high transparency [24]. It is well known that for the organic photovoltaic (OPV) device, a transparency of at least $85 \%$ and a sheet resistance of less than $15 \Omega /$ square is needed to fabricate competitive electrical devices. These wires are usually synthesized using the polyol process with

ethylene glycol as reducing agent and poly(vinylpyrrolidone) (PVP) as stabilizer [25, 26]. In this 
research, PVP is used as a stabilizer as well as growth enhancer through preferential adsorption on Ag facets leading to the unidirectional growth of the wire. Several parameters are known to affect the AgNW growth such as PVP molecular weight [27, 28], reaction temperature [28, 29], and reaction time $[28,29]$. Once synthesized, the AgNW can be deposited on a flat substrate by spin coating [30, 31], spray coating [32-35], or drop and rod casting [36, 37] yet the stability of the wire on the substrate largely depends on the types of interaction with the substrate. Glass slides which are commonly used as substrate in photovoltaic application only provide weak interactions with $\mathrm{AgNW}$ leading to poor adhesion and thus to rapid loss of conductivity during the fabrication steps of the cell due to AgNW detachment.

Polyelectrolyte multilayer (PEM) thin films fabricated using the so called layer-by-layer deposition can be used to modify the surface of glass or PET through the electrostatic or Van der Waals interactions $[38,39]$. The polyelectrolytes adhere onto the substrate through electrostatic interaction and in some case through weak Van der Waals interaction. These versatile and easy to process technique are applicable to large scale samples and environmental friendly. Since the PEM coatings are transparent ultrathin film of few tens of nanometers, they can be used as a primer to immobilize AgNW on any given substrate. Publications describing the deposition of AgNW on PEM because polyelectrolytes are generally electrical insulator which affect the electrical conductivity of AgNW thin film. Previously, PEM coating based on the assembly of Poly(allylamine hydrochloride) (PAH) and AgNW were achieved by dip coating to get films with a sheet resistance of $60 \Omega$ /square and a transparency of $78 \%$ transmission. The sheet resistance was relatively high compared to the commercial ITO coated on the glass which is around $13.6 \Omega$ /square. The lower conductivity is probably due to the insulating PAH coating in between each AgNW layers which acts as insulating barrier [40]. A more efficient approach to 
improve the conductivity is possibly to limit the AgNW coating to a single layer on top of a PEM, which is used to anchor and stabilize the wires. If successful, a standard peeling test should be used to confirm the anchoring and stability of the silver wires on the PEM substrate while maintaining good transparency and good conductivity.

In this paper, robust transparent conducting electrodes were prepared by spin coating of PVP stabilized AgNW on a PEM primer layer coated on a glass substrate. To investigate the effect of the top layer charge, PEM composed of 7 (positively charged on top) or 8 layers (negatively charged on top) were used as adhesive layer to anchor the AgNW. The conductivity and transparency of the different coating was compared using 4 points probe measurements before and after up to 6 peeling test cycles. Finally, the AgNW were also deposited on a poly(ethylene terephthalate) (PET) substrate to fabricate flexible TCE, to be used in flexible solar cell application.

\section{Experimental}

\subsection{Material}

Silver nitrate $\left(\mathrm{AgNO}_{3}, \mathrm{ACS}\right.$ reagent, $\left.>99.0 \%\right)$, glycerol (>99.5\%), poly(diallyl dimethyl ammonium chloride) solution (PDADMAC, medium molecular weight, 20 wt. $\%$ in $\mathrm{H}_{2} \mathrm{O}$, MW 200,000-350,000), poly(sodium 4-styrenesulfonic acid) sodium salt (PSS, average MW 70,000), sodium chloride $(\mathrm{NaCl}$, ACS reagent, $>99.0 \%)$ were purchased from Sigma-Aldrich. Poly(vinylpyrrolidone) (PVP, M.W. 1,300,000) was purchased from Alfa Aesar. Absolute ethanol (EMPROVE ${ }^{\circledR}$ ) were purchased from Merck. All chemicals were analytical grade and used without further purification. 


\subsection{Synthesis of AgNW}

The AgNW were synthesized using a modified solvothermal method in which ultra-long silver nanowires of over $20 \mu \mathrm{m}$ could be produced [41]. In this method $2.553 \mathrm{~g}$ of PVP were dissolved in $70 \mathrm{~mL}$ of glycerol under stirring at $100^{\circ} \mathrm{C}$ for 8 hours. In the meantime, $0.51 \mathrm{~g}$ of $\mathrm{AgNO}_{3}$ and $3.62 \mathrm{mg}$ of $\mathrm{NaCl}$ were dissolved in $10 \mathrm{~mL}$ and $20 \mathrm{~mL}$ of glycerol, respectively. The $\mathrm{NaCl}$ solution was stirred and heated at $50^{\circ} \mathrm{C}$ in order to obtain the clear solution. Then, $\mathrm{AgNO}_{3}$ and $\mathrm{NaCl}$ were added into PVP solution and stirred for 3 minutes at $100^{\circ} \mathrm{C}$, the solution changed from transparent to orange which confirmed the AgNP seed growth. The mixed solution was instantly transferred to the Teflon-lined stainless steel autoclave and heat at $150^{\circ} \mathrm{C}$ for 5.5 hours for the growing of AgNW. The AgNW was purified by washing with the mixed solvent between deionized water and absolute ethanol (ratio 1:1) several times and centrifuged at 4,500 rpm for 10 min. Finally, AgNW was washing with ethanol 3 more times and re-dispersed in ethanol for further used.

\subsection{PEM fabrication}

PEM were fabricated on a glass substrate which was sequentially sonicated in DI water, acetone, isopropanol, and dried with Argon. The glass was then treated under UV light for 15 min. The treated glass was alternatively immersed for $1 \mathrm{~min}$ into $10 \mathrm{mM}$ of PDADMAC solution and $10 \mathrm{mM}$ of PSS with $1 \mathrm{M} \mathrm{NaCl}$. After each polyelectrolyte immersion steps, the excess PDADMAC or PSS was removed by rinsing in DI water $30 \mathrm{sec}$ for 3 times. This dipping cycle was repeated until the desired primer was achieved, then it was dried with Argon. AgNW were deposited on the glass, PEM 7 layers, and PEM 8 layers substrate by spin coating with a speed of 
$1,000 \mathrm{rpm}$ for $1 \mathrm{~min}$. The substrates were next post-annealed at $150^{\circ} \mathrm{C}$ for $10 \mathrm{~min}$ in order to dry the films and improve the electrical properties of AgNW network on the substrate.

\subsection{Characterization}

UV-Visible spectroscopy (Jasco, V-670 spectrometer with FLH-740 head) was used to observe the optical properties of AgNW and also the transparency which was measured at 550 nm. X-Ray diffraction spectroscopy (XRD, Rigaku SmartLab, Japan) was used to record the Xray diffraction pattern of $\mathrm{AgNW}$ using $\mathrm{Cu} \mathrm{K} \alpha$ radiation at room temperature with scan rate of $2 \theta$ $=2^{\circ} / \mathrm{min}$ and scanning from $30-90^{\circ}$. Field emission scanning electron microscope (FESEM, S4800, Hitachi) was used to observe the size, shape and morphology of AgNW on the specimen's surface. The peeling resistance was observed by using the captain scotch tape (927AR grade) with thickness of $0.055 \mathrm{~mm}$. Atomic force microscopy (AFM, Park system XE-Series, noncontact mode) was used to observe the surface topology of the AgNW on the substrate. The sheet resistance was measured with a 4 point probe setup (Lucas labs model 302) connected to a Keithley 236 source measure unit. The distance between each probe was $0.5 \mathrm{~mm}$. The glass specimen was cut to $2 \mathrm{~cm} \times 2.5 \mathrm{~cm}$ and coated with PEM followed by AgNW deposition via spin coating.

\section{Result and discussion}

The AgNW were synthesized using the solvothermal process with PVP and glycerol as stabilizer and reducing agent. The synthesis was carried out with $\mathrm{NaCl}$ in solution leading to the formation of $\mathrm{AgCl}$ complex which was later reduced to silver $\mathrm{Ag}^{0}$ and obtained uniform and long AgNW. Although, ethylene glycol is a common reducing agent in the synthesis of AgNW, 
glycerol was used here as an alternative reducing agent. Glycerol has a higher boiling point (290 ${ }^{\circ} \mathrm{C}$ ) and a higher hydroxyl group content than ethylene glycol which accelerated the wires growth $[26,42]$. As in other published work, PVP was used to control the growth direction of AgNW as it present favorable adsorption onto the (100) face of the multiple-twined particle of silver. This preferential adsorption leaves the (111) face unprotected, thus inducing a longitudinal growth of the wire perpendicular to the (111) direction. A monolayer of AgNW was then spin-coated on the PEM primer which acted as the adhesive layer as shown in Fig.1. The PEM top layer is expected to provide an anchoring site for the AgNW and possibly to enhance the peeling resistivity and also the AgNW packing density on the substrate when compared to deposition on bare glass surface.

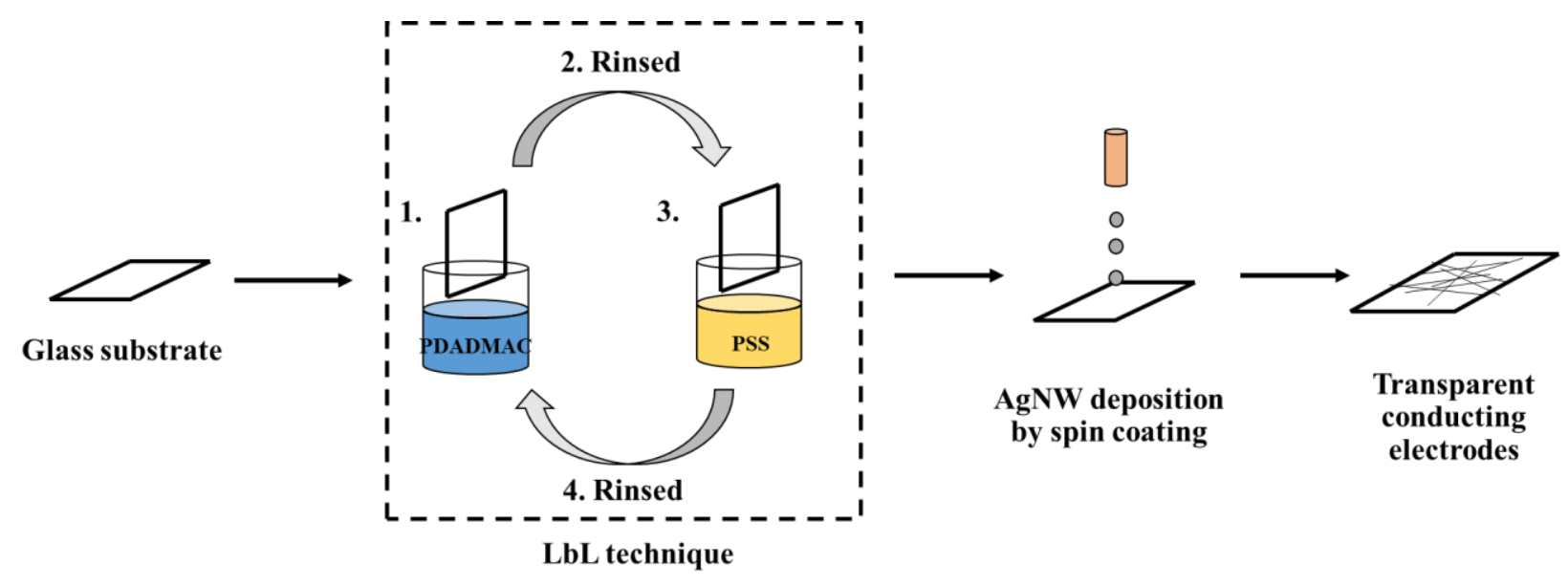

Fig. 1. Schematic of the method used for the silver nanowires deposition

Electron microscopy was used to observe the morphology of the wire which should display nanometer scale diameter but micron scale length. On the FESEM images of AgNW shown in Fig.2, it can be seen that the diameter of the AgNW is between 20 to $30 \mathrm{~nm}$ with a length ranging from 10 to $30 \mu \mathrm{m}$ confirming the successful synthesis of wires. Typically, spherical silver nanoparticles usually display an absorbance peak centered around $400 \mathrm{~nm}$, 
depending on size, shape and surrounding media. This plasmonic peak is due to the collective oscillation of the delocalized free electron in the metallic nanoparticle under electro-magnetic excitation. From the UV absorption spectrum of AgNW, two peaks are usually observed in the literature [27] at 350 and $380 \mathrm{~nm}$, which are characteristic of the AgNW UV-Vis spectra. In Fig.2, these two peaks are clearly visible and no peak can be seen after $400 \mathrm{~nm}$ suggesting that all the silver nanoparticles have been consumed and transformed to silver wires. If the silver nanoparticles were only converted to short silver rods having small aspect ratio (10-50), they would present a longitudinal resonance plasmon peak shifted to higher wavelength in the visible range. Here, the wires with an aspect ratio of nearly 1,000 only display transverse plasmon band and their secondary peak is not visible. Both UV absorption spectra and FESEM image confirm that no silver nanoparticle seed are left after completion of the reaction and that the $\mathrm{AgNW}$ with a diameter of 20-30 nm and a length of around 20-30 $\mu \mathrm{m}$ have been synthesized.
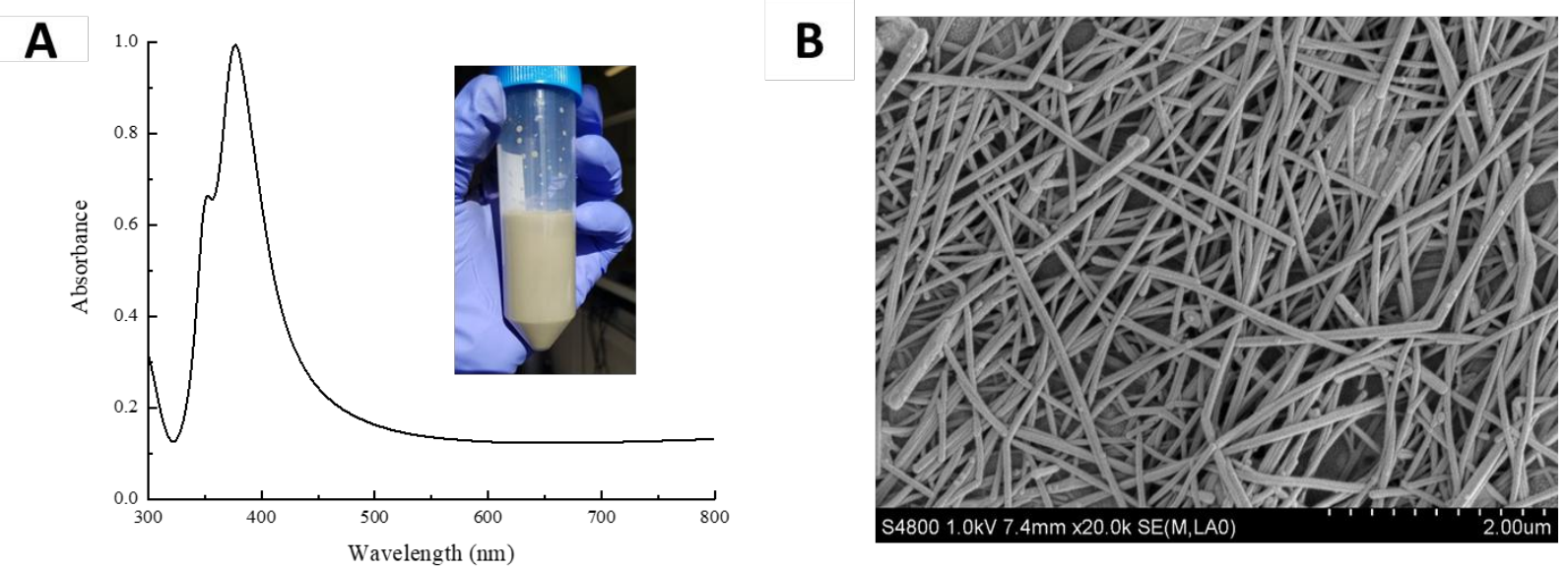

Fig. 2. UV-Vis absorption spectra and pictures of the AgNW solution (A) and FESEM image of $\mathrm{AgNW}(\mathrm{B})$ 
The crystal structure of the AgNW was studied by X-Ray Diffraction pattern analysis. As the wires grow in the (111) plane direction, this lattice should be dominant in the asymmetric growth of the silver crystal. The XRD analysis of the sample shown in Fig.3 display the characteristic diffraction pattern of the face centered cubic of AgNW which consists of two main diffraction peaks at $38.3^{\circ}$ and $44.7^{\circ}$. These two largely predominant peaks that correspond to the (111) and (200) diffraction planes hinder the observation of the (220) and (311) that should appear at $64.9^{\circ}$ and $78.1^{\circ}$, as suggested by Shobin et al $[27,43]$.

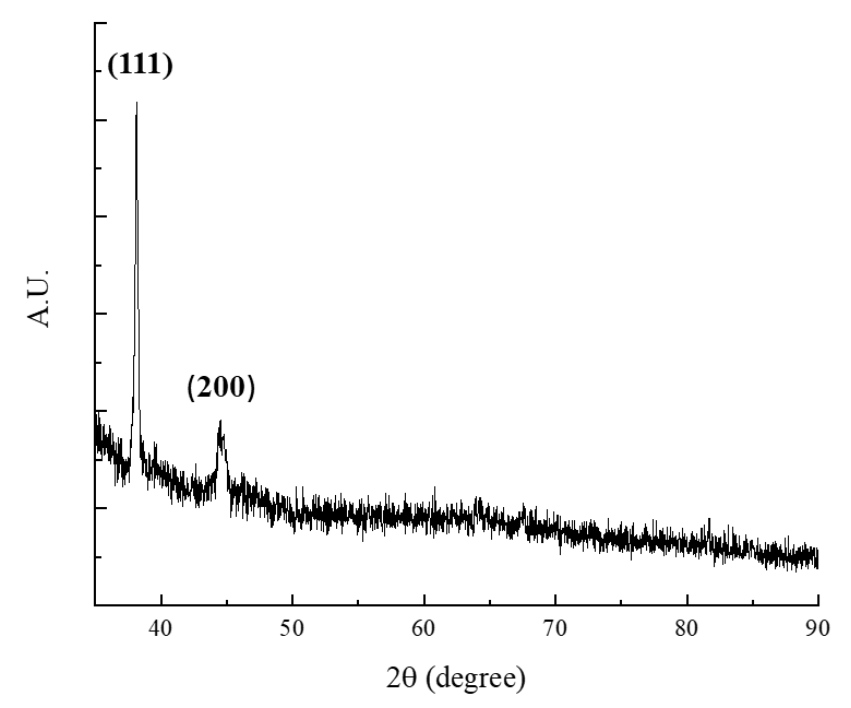

Fig. 3. Plot of the XRD pattern from a thin film of AgNW coated on a glass substrate

It was initially hypothesized that the PEM coatings could enhance the deposition and stability of AgNW on glass slides. To test this hypothesis, a PEM primer layer was deposited on glass slides with 7 or 8 layers to provide either a cationic or anionic top surface charge. PVP is a non-ionic polymer used to stabilize the AgNW and its possible interaction with the PEM was tested on both charge surfaces. The AgNW were deposited by spin coating on bare glass slide 
and each 7 or 8 layers PEM. The surface of the samples after spin coating was analyzed using AFM to study theirs topology and surface roughness (Fig.4).
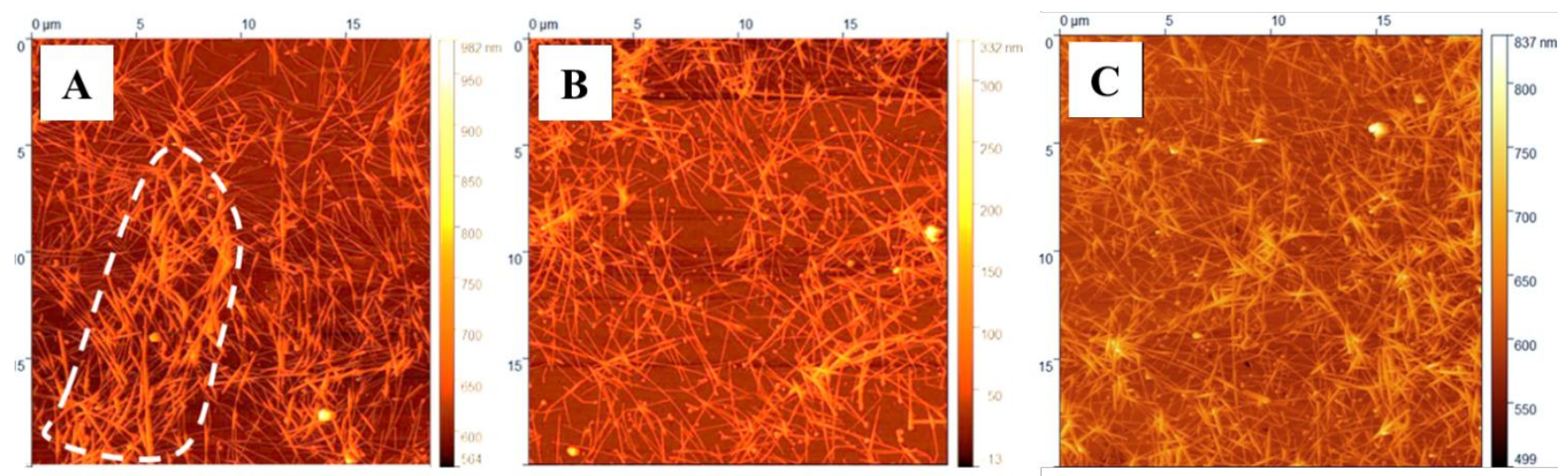

Fig. 4. AFM images of the AgNW coated on (A) bare glass, (B) glass slide with 8 PDADMACPSS layers and (C) glass slide with 7 PDADMAC-PSS layers

From the AFM images of all the samples, it can be seen that the AgNW are individually deposited and no aggregation of single AgNW into bundles is observable. The diameter measured using the AFM by cross section analysis confirm a diameter of $20-30 \mathrm{~nm}$ which is consistent with the FESEM images in Fig 2. It can be seen that the AgNW deposited on the glass substrate (Fig 4A) appear partially aggregated into clusters, suggesting a less uniform coating with an average roughness of $28.87 \mathrm{~nm}$. The aggregation of the wires is probably due to the residual mobility of the wires during solvent evaporation due to poor AgNW-glass interaction. The anionic PEM 8 layers coated with the AgNW displayed a more homogeneous dispersion due to the better anchoring, but the wires density appear lower which might be due to the repulsive force between anionic PSS and carbonyl groups found on the PVP capping. Interestingly, the cationic PEM 7 layers displayed a more homogeneous and higher density coating as seen in Figure 4C. The surface roughness of AgNW on PEM 7 and 8 layers were measured to be 28.02 and $23.9 \mathrm{~nm}$, respectively. The AgNW coating density can also be evaluated by using UV-Vis 
spectroscopy, which is proportional to the AgNW local density. In Fig. 5, the absorbance values for each coating are compiled and it can be seen that PEM 7 layers coating has a higher absorbance at 350 and $380 \mathrm{~nm}$ when compared to PEM 8 layers and bare glass slide, thus confirming AFM images.

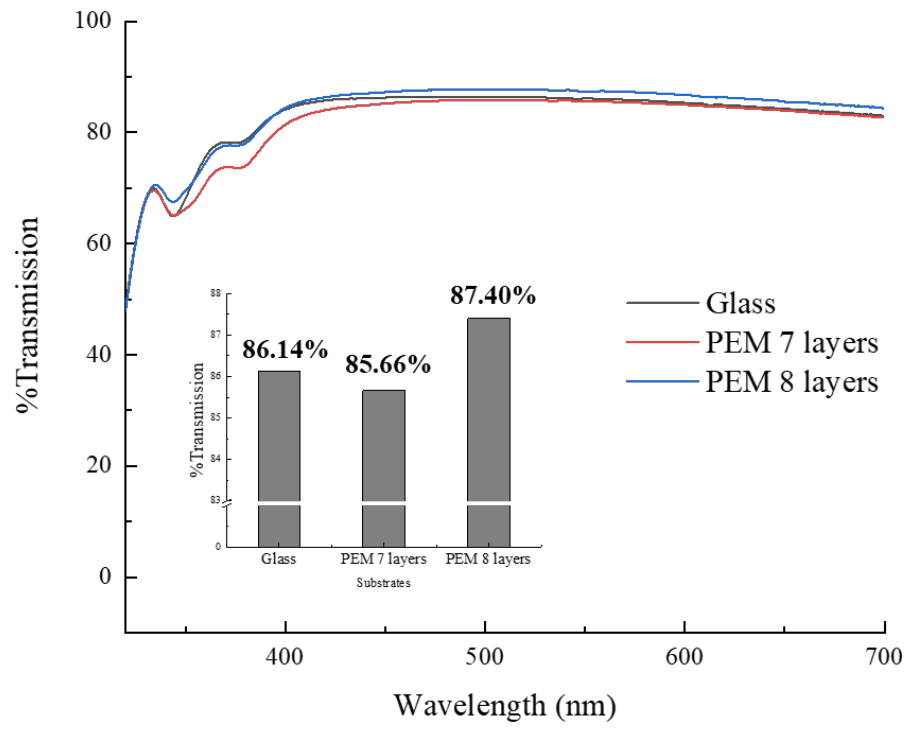

Fig. 5. UV-Vis transmission spectra of the $\mathrm{AgNW}(0.7 \% \mathrm{w} / \mathrm{v})$ coated on glass and 7 or 8 layers PEM. The inset represents the \%transmission values at $550 \mathrm{~nm}$ for each samples 


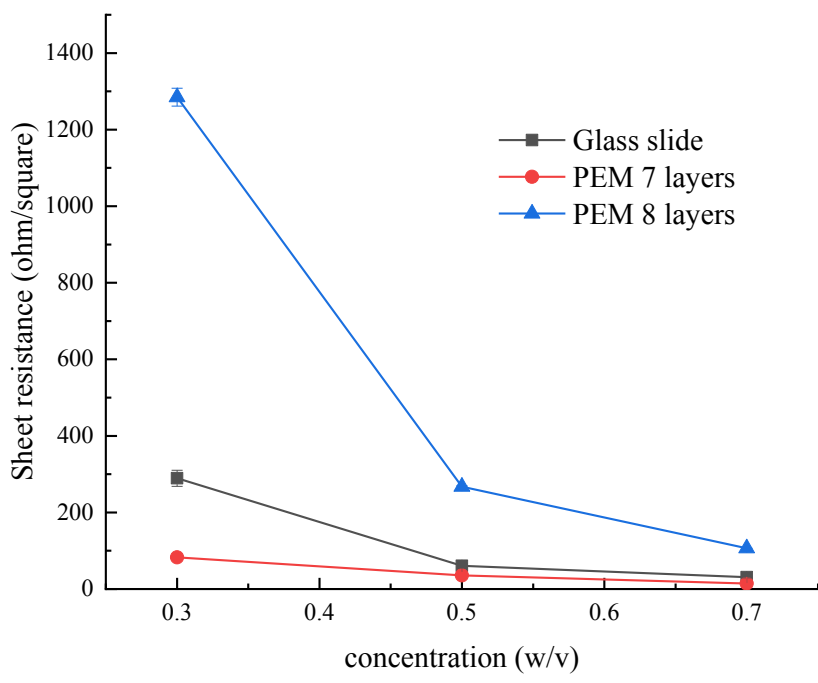

Fig. 6. Plot of the sheet resistance of the AgNW coating on bare glass, 7 and 8 layers PDADMAC-PSS layers as a function of the concentration of $\mathrm{AgNW}(\% \mathrm{w} / \mathrm{v})$ in solution

With transparency, the other most critical characteristic of the AgNW coating is its electrical conductivity. In Fig.6, the sheet resistance of samples prepared from increasing AgNW content and spin-coated on bare glass slides and PEM 7 or 8 layers is shown. Using the 4-points probe technique to evaluate the conducting properties of the different coating, the sheet resistances of the AgNW (0.7 \%wt.) coated on glass and PEM 8 layers were measured to be 30 and $106 \Omega$ /square, respectively (Fig.6). The AgNW coated on PEM 7 layers had sheet resistance as low as $14 \Omega$ /square. It can also be seen that the sheet resistance decreased with the increasing AgNW concentration which is fairly intuitive. Nevertheless, the 7 layers coating produced a denser and more homogeneous AgNW coating with the best conductivity when compared to blank and 8 layers coating. Although, AgNW and PVP do not carry a clear anionic charge, favorable interaction between the lone pair on the $\mathrm{N}$ and PDADMAC coating are expected. Similar affinity between $\mathrm{N}$ atom in PAH and the AgNW have previously been reported by Kim 
et al [40]. They used XPS was used to confirm the formation of coordinating bond between the nitrogen atom and the AgNW surface. On the other hand, both glass and PEM 8 layers substrate display higher sheet resistance suggesting aggregation and poor coating density. Although all samples could be coated by spin coating with more or less success, it is necessary to investigate the stability of the wire on the surface with a peeling test 6 cycles of pressing a scotch tape strip on different samples followed by quick peeling.

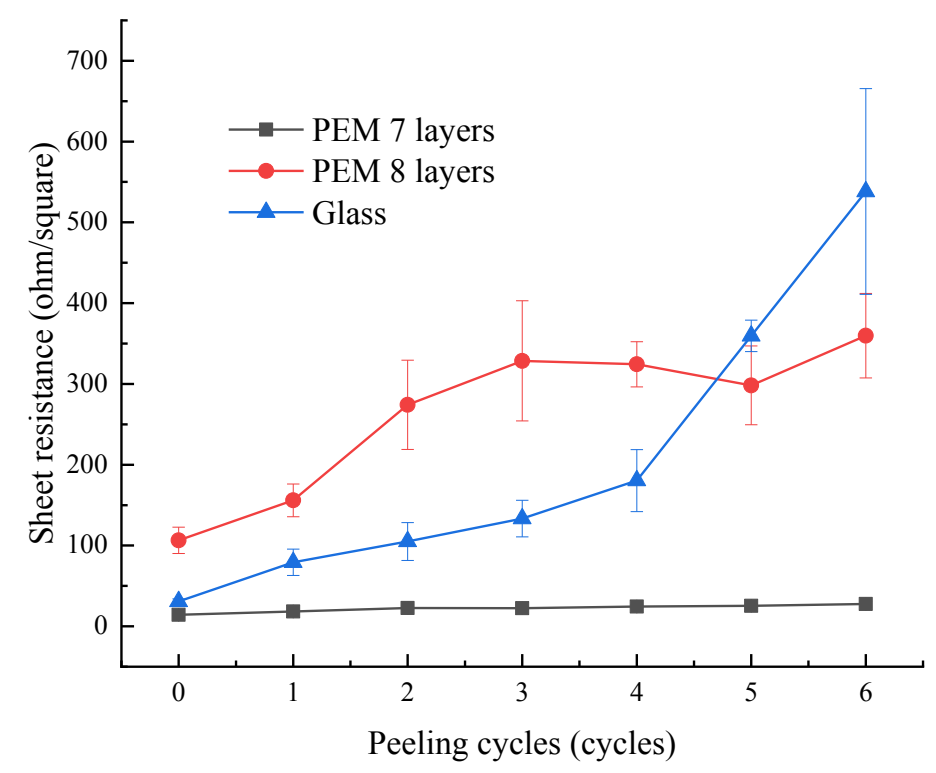

Fig. 7. Plot of the sheet resistance of the AgNW coated on glass, PEM 7 and 8 layers as a function of the number of peeling cycle

As displayed in Fig.7, the sheet resistance of AgNW coating was measured after each cycles and was found to increase in the case of the glass coating and PEM 8 layers from 30 to about $500 \Omega$ /square after 6 peeling cycles suggesting poor adhesion of both coating. In contrast, the AgNW coated on the PDADMAC (PEM 7 layers) displayed a much better adhesion and 
stability and maintained a sheet resistance value under $25 \Omega /$ square even after 6 cycles of peeling.

It can be clearly concluded that favorable interaction between the wires and the PDADMAC layers lead to a better adhesion properties using the PEM 7 layers. As the ultimate goal is to produce flexible transparent conducting electrode, a bare PET sample was used as substrate while two other PET samples were modified with 7 and 8 layer of PDADMAC-PSS which were used as adhesive layer for AgNW. The sheet resistance of all samples after coating with AgNW were measured and plotted in Fig.8.

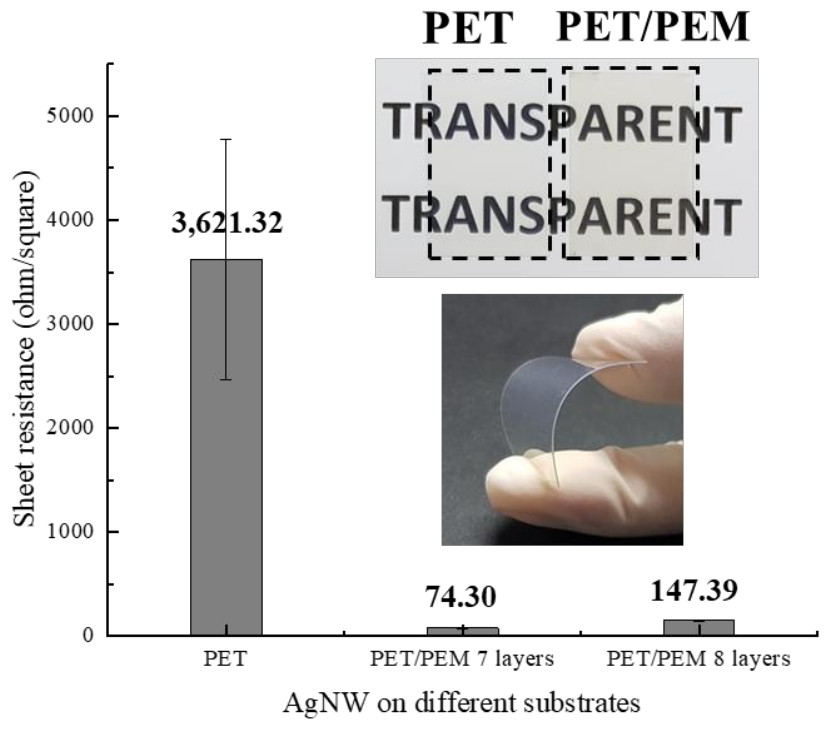

Fig. 8. Sheet resistance of AgNW deposited on the PET and PET/PEM 7 and 8 layers. Pictures of the bare and 7 layers coated PET coated (Upper picture) and 7 layers coated PET (lower picture)

Results show that the sheet resistance of the AgNW on PET is significantly lower than on glass different ( $30 \Omega /$ square for glass and 3,600 $\Omega /$ square for PET) due to the hydrophilic characteristic of PET which affect to the binding of the AgNW and also the fabrication of PEM. 
By using the PEM 7 and 8 layers as adhesive layer, the PET surface became more hydrophilic which provided a better deposition of AgNW result in the lower sheet resistance. Similarly to the deposition of AgNW on the glass substrate, the PEM 7 layers exhibited a better adhesion of AgNW than the PEM 8 layers while maintaining a good transparency when compared to the plain PET without AgNW (Fig.9A). It can be concluded that the modification of the PET substrate with PEM 7 layers produced flexible (Fig.8) and transparent PET (95\% transmission) while maintaining good conductivity ( $74 \Omega /$ square)

\section{Conclusion}

In this work, transparent conducting electrodes were prepared by the deposition of AgNW on PEM modified glass substrates via spin coating. The spin coating of the AgNW on all three surfaces (bare, 7 and 8 layer PDADMAC-PSS) was successful although a better deposition of the AgNW was obtained when PDADMAC was used as top layer (7 layers) in term of coating density (UV-Vis) and dispersion (4 point probe). Additionally, the PDADMAC could prevent the peeling of the AgNW and maintain a low sheet resistance even after 6 peeling cycles. PET was also used as substrate for the deposition of $\mathrm{AgNW}$ in order to produce the flexible transparent conducting electrode which could be used in solar cell application. Results suggest that the treatment of the PET substrates, using a PEM primer with PDADMAC on top is a promising method to stabilize AgNW toward the development of robust transparent conducting electrodes for the development of optoelectronic devices.

\section{Acknowledgement}


This work was supported by the Thailand research fund [PHD59I0034, 2015]; and Centre interdisciplinaire de nanoscience de Marseille, CINaM, Marseille.

\section{Reference}

[1] T.-B. Song, N. Li, Emerging Transparent Conducting Electrodes for Organic Light Emitting Diodes, Electronics, 3 (2014) 190-204.

[2] A. Kumar, Predicting efficiency of solar cell based on transparent conducting electrode, Journal of Applied Physics, 121 (2017).

[3] R. Peng, W. Song, T. Yan, B. Fanady, Y. Li, Q. Zhan, Z. Ge, Interface bonding engineering of a transparent conductive electrode towards highly efficient and mechanically flexible ITO-free organic solar cells, Journal of Materials Chemistry A, 7 (2019) 11460-11467.

[4] J. Meiss, M.K. Riede, K. Leo, Towards efficient tin-doped indium oxide (ITO)-free inverted organic solar cells using metal cathodes, Applied Physics Letters, 94 (2009).

[5] H. Makino, Yamamoto, N., Miyake, A., Yamada, T., Iwaoka, H., P-7: Ga-Doped ZnO Transparent Conductive Film as Substitution for ITO Common Electrode in TFT-LCDs, SID Symposium DIgest of Technical Papers, 40 (2009) 1103-1106.

[6] N.M. Bedford, M.B. Dickerson, L.F. Drummy, H. Koerner, K.M. Singh, M.C. Vasudev, M.F. Durstock, R.R. Naik, A.J. Steckl, Nanofiber-Based Bulk-Heterojunction Organic Solar Cells Using Coaxial Electrospinning, Advanced Energy Materials, 2 (2012) 1136-1144.

[7] T.-H. Han, S.-H. Jeong, Y. Lee, H.-K. Seo, S.-J. Kwon, M.-H. Park, T.-W. Lee, Flexible transparent electrodes for organic light-emitting diodes, Journal of Information Display, 16 (2015) 71-84. 
[8] S.W. Shin, Y.U. Jung, K.-B. Kim, S.-W. Choi, S.J. Kang, ITO-free transparent conductive films based on carbon nanomaterials with metal grid for liquid crystal displays, Liquid Crystals, $42(2015)$ 954-958.

[9] D. Kim, L. Zhu, D.-J. Jeong, K. Chun, Y.-Y. Bang, S.-R. Kim, J.-H. Kim, S.-K. Oh, Transparent flexible heater based on hybrid of carbon nanotubes and silver nanowires, Carbon, 63 (2013) 530-536.

[10] K. Sakamoto, H. Kuwae, N. Kobayashi, A. Nobori, S. Shoji, J. Mizuno, Highly flexible transparent electrodes based on mesh-patterned rigid indium tin oxide, Sci Rep, 8 (2018) 2825.

[11] P.C. Hsu, S. Wang, H. Wu, V.K. Narasimhan, D. Kong, H. Ryoung Lee, Y. Cui, Performance enhancement of metal nanowire transparent conducting electrodes by mesoscale metal wires, Nat Commun, 4 (2013) 2522.

[12] M. Arefpour, M. Almasi Kashi, F. Khansari Barzoki, M. Noormohammadi, A. Ramazani, Electrodeposited metal nanowires as transparent conductive electrodes: Their release conditions, electrical conductivity, optical transparency and chemical stability, Materials \& Design, 157 (2018) 326-336.

[13] C.F. Guo, Z. Ren, Flexible transparent conductors based on metal nanowire networks, Materials Today, 18 (2015) 143-154.

[14] R. Zhang, M. Engholm, Recent Progress on the Fabrication and Properties of Silver Nanowire-Based Transparent Electrodes, Nanomaterials (Basel), 8 (2018).

[15] Z.C. Zhuangchun Wu, Xu Du, Jonathan M. Logan, Jenifer Sippel, Maria Nikolou, Katalin Kamaras, John R. Reynolds, David B. Tanner, Arthur F. Hebard, Andrew G. Rinzler, Transparent, Conductive Carbon Nanotube Films, Science, 205 (2004) 1273-1276. 
[16] O. Urper, İ. Çakmak, N. Karatepe, Fabrication of carbon nanotube transparent conductive films by vacuum filtration method, Materials Letters, 223 (2018) 210-214.

[17] Y.S. Woo, Transparent Conductive Electrodes Based on Graphene-Related Materials, Micromachines (Basel), 10 (2018).

[18] C. Guillén, J. Herrero, Transparent conductive ITO/Ag/ITO multilayer electrodes deposited by sputtering at room temperature, Optics Communications, 282 (2009) 574-578.

[19] M.A. Shinde, K. Mallikarjuna, J. Noh, H. Kim, Highly stable silver nanowires based bilayered flexible transparent conductive electrode, Thin Solid Films, 660 (2018) 447-454.

[20] M.A. Shinde, D.-J. Lee, B.-J. Kim, H. Kim, Highly conductive and smooth surfaced flexible transparent conductive electrode based on silver nanowires, Thin Solid Films, 685 (2019) 366371.

[21] A.H. Alami, B. Rajab, K. Aokal, Assessment of silver nanowires infused with zinc oxide as a transparent electrode for dye-sensitized solar cell applications, Energy, 139 (2017) 1231-1236.

[22] M. Singh, P. Prasher, J. Kim, Solution processed silver-nanowire/zinc oxide based transparent conductive electrode for efficient photovoltaic performance, Nano-Structures \& Nano-Objects, 16 (2018) 151-155.

[23] M. Chalh, S. Vedraine, B. Lucas, B. Ratier, Plasmonic Ag nanowire network embedded in zinc oxide nanoparticles for inverted organic solar cells electrode, Solar Energy Materials and Solar Cells, 152 (2016) 34-41.

[24] T. Tokuno, M. Nogi, M. Karakawa, J. Jiu, T.T. Nge, Y. Aso, K. Suganuma, Fabrication of silver nanowire transparent electrodes at room temperature, Nano Research, 4 (2011) 1215-1222. 
[25] P. Zhang, I. Wyman, J. Hu, S. Lin, Z. Zhong, Y. Tu, Z. Huang, Y. Wei, Silver nanowires: Synthesis technologies, growth mechanism and multifunctional applications, Materials Science and Engineering: B, 223 (2017) 1-23.

[26] B. Liu, H. Yan, S. Chen, Y. Guan, G. Wu, R. Jin, L. Li, Stable and Controllable Synthesis of Silver Nanowires for Transparent Conducting Film, Nanoscale Res Lett, 12 (2017) 212.

[27] S. Coskun, B. Aksoy, H.E. Unalan, Polyol Synthesis of Silver Nanowires: An Extensive Parametric Study, Crystal Growth \& Design, 11 (2011) 4963-4969.

[28] Y. Ran, W. He, K. Wang, S. Ji, C. Ye, A one-step route to Ag nanowires with a diameter below $40 \mathrm{~nm}$ and an aspect ratio above 1000, Chem Commun (Camb), 50 (2014) 14877-14880.

[29] H.-W. Jang, B.-Y. Hwang, K.-W. Lee, Y.-M. Kim, J.-Y. Kim, Controlling the size of silver nanowires produced by a tetrabutylammonium dichlorobromide salt-based polyol process: Kinetics of silver crystal growth, AIP Advances, 8 (2018).

[30] H. Wang, Y. Wang, X. Chen, Synthesis of uniform silver nanowires from $\mathrm{AgCl}$ seeds for transparent conductive films via spin-coating at variable spin-speed, Colloids and Surfaces A: Physicochemical and Engineering Aspects, 565 (2019) 154-161.

[31] Y. Ko, J. Kim, D. Kim, Y. Yamauchi, J.H. Kim, J. You, A Simple Silver Nanowire Patterning Method Based on Poly(Ethylene Glycol) Photolithography and Its Application for Soft Electronics, Sci Rep, 7 (2017) 2282.

[32] H. Yun, D. Seo, M. Lee, S. Kwon, L. Park, Effective Synthesis and Recovery of Silver Nanowires Prepared by Tapered Continuous Flow Reactor for Flexible and Transparent Conducting Electrode, Metals, 6 (2016).

[33] J.-Y. Lee, D. Shin, J. Park, Fabrication of silver nanowire-based stretchable electrodes using spray coating, Thin Solid Films, 608 (2016) 34-43. 
[34] F. Selzer, N. Weiss, D. Kneppe, L. Bormann, C. Sachse, N. Gaponik, A. Eychmuller, K. Leo, L. Muller-Meskamp, A spray-coating process for highly conductive silver nanowire networks as the transparent top-electrode for small molecule organic photovoltaics, Nanoscale, 7 (2015) 2777-2783.

[35] J.S. Woo, G.-W. Lee, S.-Y. Park, J.T. Han, Realization of transparent conducting networks with high uniformity by spray deposition on flexible substrates, Thin Solid Films, 638 (2017) 367-374.

[36] C. Sachse, L. Müller-Meskamp, L. Bormann, Y.H. Kim, F. Lehnert, A. Philipp, B. Beyer, K. Leo, Transparent, dip-coated silver nanowire electrodes for small molecule organic solar cells, Organic Electronics, 14 (2013) 143-148.

[37] Junaidi, T. K., Harsojo, S. E., High-Performance Silver Nanowire Film on Flexible Substrate Prepared by Meyer-rod Coating, Materials Science and Engineering: B, 202 (2017).

[38] S.T. Dubas, J.B. Schlenoff, Factors Controlling the Growth of Polyelectrolyte Multilayers, Macromolecules, 32 (1999) 8153-8160.

[39] S.T. Dubas, J.B. Schlenoff, Swelling and Smoothing of Polyelectrolyte Multilayers by Salt, Langmuir, 17 (2001) 7725-7727.

[40] C. Kim, H. An, A. Jung, B. Yeom, Vortex-assisted layer-by-layer assembly of silver nanowire thin films for flexible and transparent conductive electrodes, J Colloid Interface Sci, 493 (2017) 371-377.

[41] Y. Li, S. Guo, H. Yang, Y. Chao, S. Jiang, C. Wang, One-step synthesis of ultra-long silver nanowires of over $100 \mu \mathrm{m}$ and their application in flexible transparent conductive films, RSC Advances, 8 (2018) 8057-8063. 
[42] C. Yang, Y. Tang, Z. Su, Z. Zhang, C. Fang, Preparation of Silver Nanowires via a Rapid, Scalable and Green Pathway, Journal of Materials Science \& Technology, 31 (2014).

[43] L.R. Shobin, S. Manivannan, One pot rapid synthesis of silver nanowires using $\mathrm{NaCl}$ assisted glycerol mediated polyol process, Electronic Materials Letters, 10 (2014) 1027-1031. 


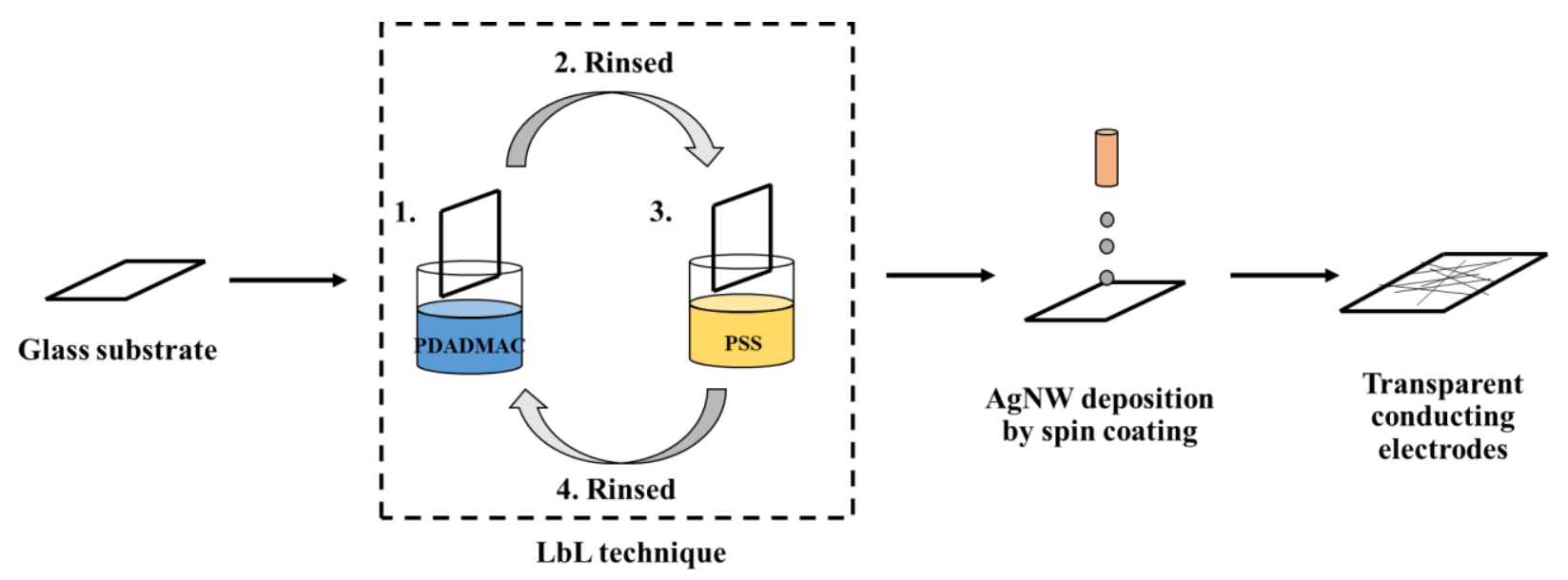

Fig. 1. Schematic of the method used for the silver nanowires deposition 
Figures (if any)

Click here to download Figures (if any): Figure 2.docx
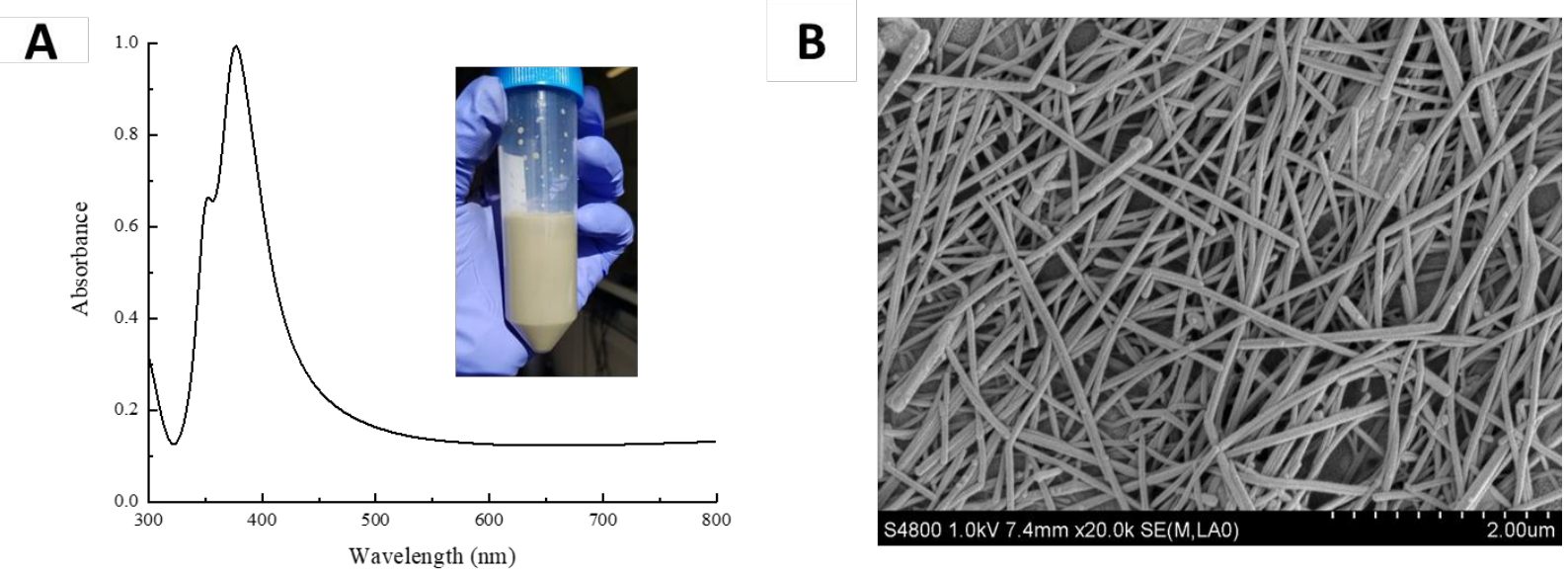

Fig. 2. UV-Vis absorption spectra and pictures of the AgNW solution (A) and FESEM image of $\operatorname{AgNW}(\mathrm{B})$ 
Click here to download Figures (if any): Figure 3.docx

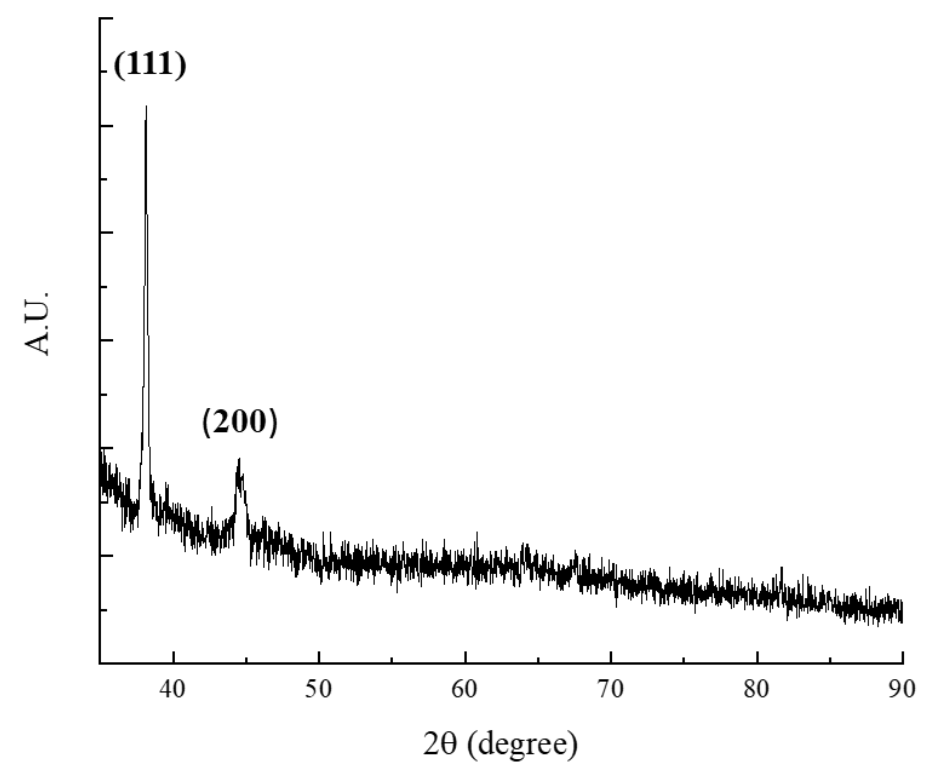

Fig. 3. Plot of the XRD pattern from a thin film of AgNW coated on a glass substrate 

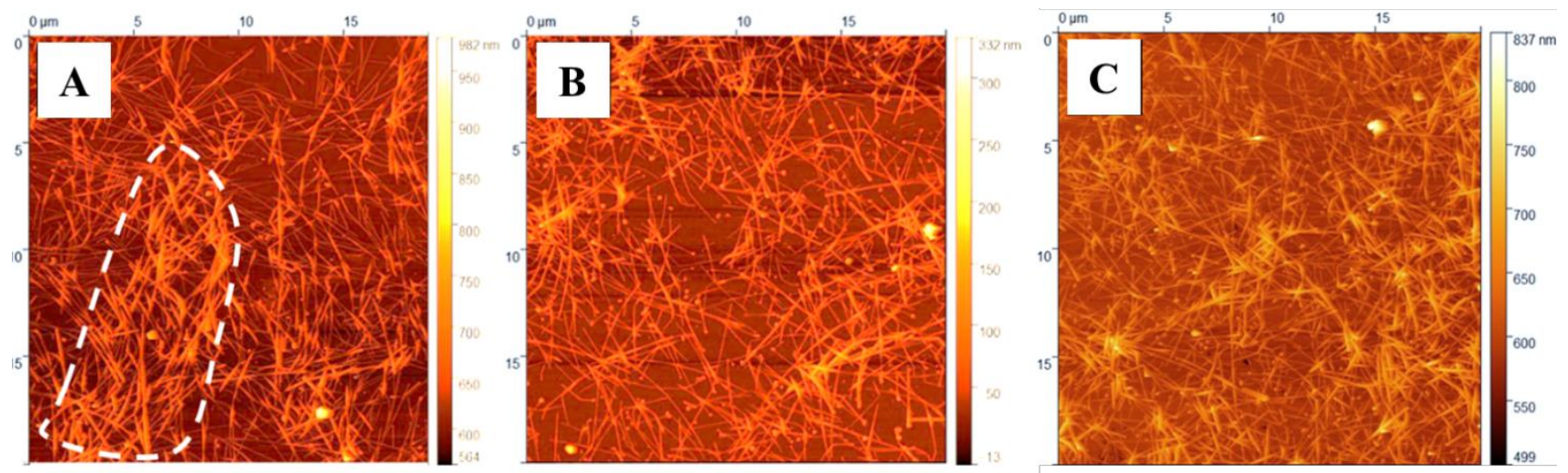

Fig. 4. AFM images of the AgNW coated on (A) bare glass, (B) glass slide with 8 PDADMACPSS layers and (C) glass slide with 7 PDADMAC-PSS layers 


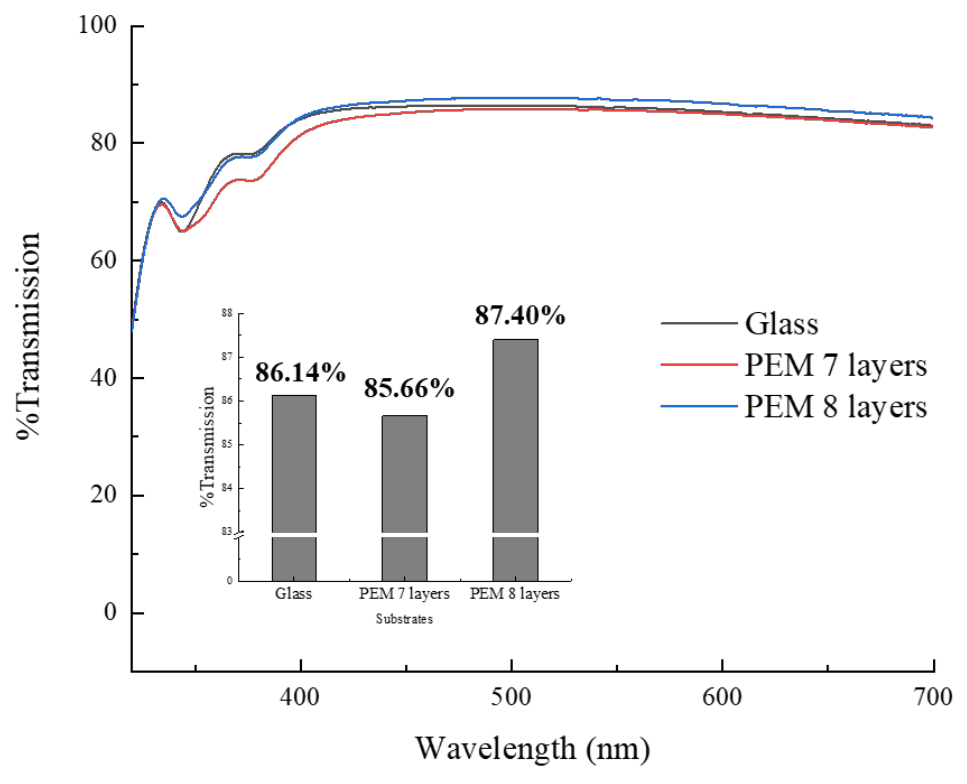

Fig. 5. UV-Vis transmission spectra of the $\mathrm{AgNW}(0.7 \% \mathrm{w} / \mathrm{v})$ coated on glass and 7 or 8 layers PEM. The inset represents the \%transmission values at $550 \mathrm{~nm}$ for each samples 


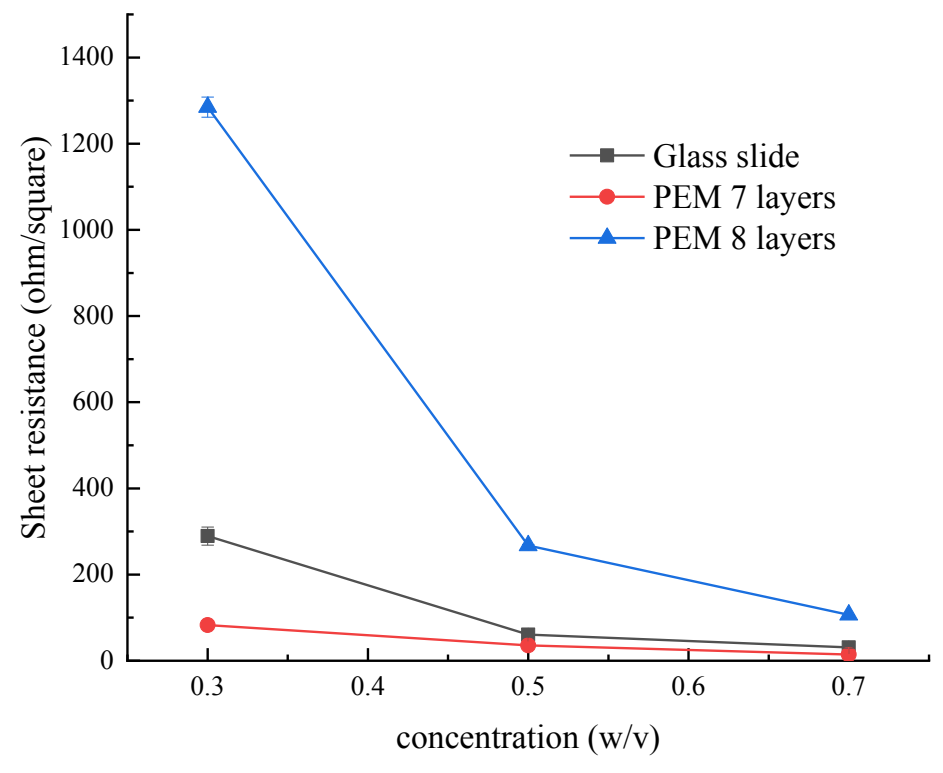

Fig. 6. Plot of the sheet resistance of the AgNW coating on bare glass, 7 and 8 layers PDADMAC-PSS layers as a function of the concentration of $\mathrm{AgNW}(\% \mathrm{w} / \mathrm{v})$ in solution 


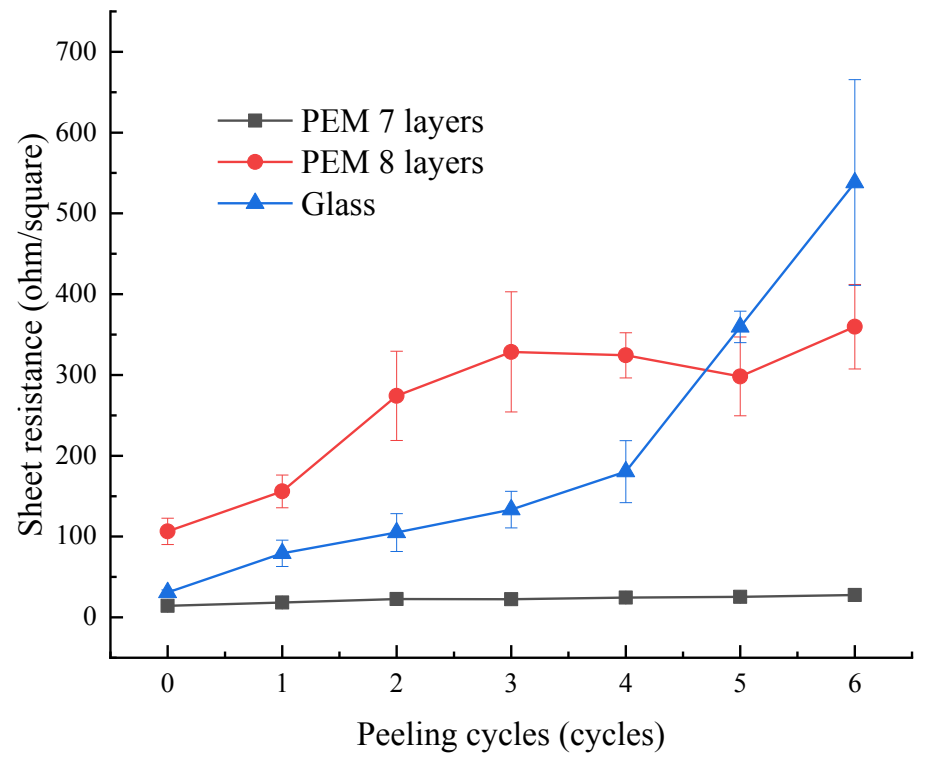

Fig. 7. Plot of the sheet resistance of the AgNW coated on glass, PEM 7 and 8 layers as a function of the number of peeling cycle 


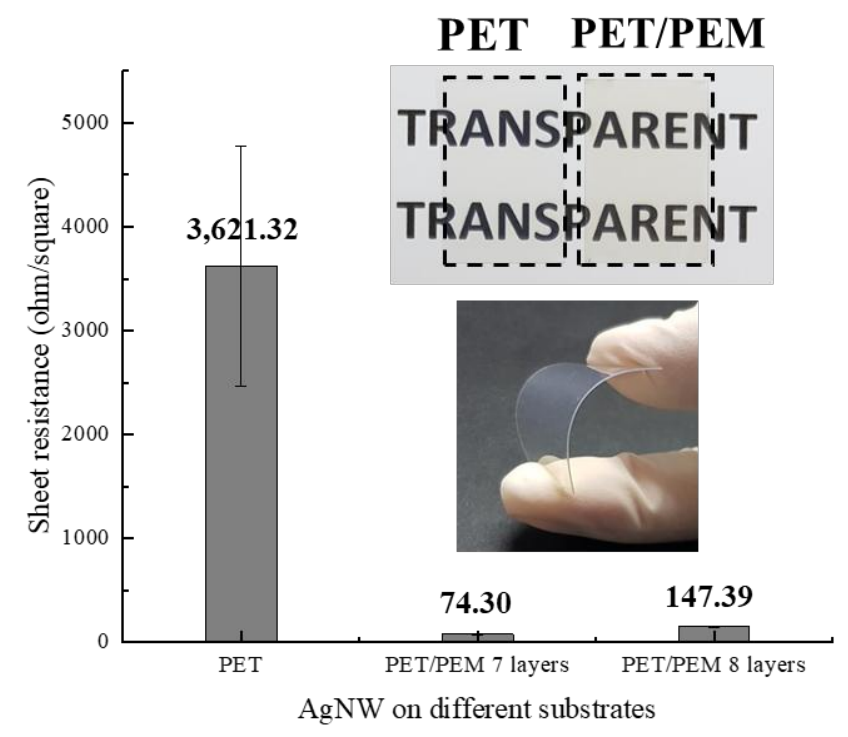

Fig. 8. Sheet resistance of AgNW deposited on the PET and PET/PEM 7 and 8 layers. Pictures of the bare and 7 layers coated PET coated (Upper picture) and 7 layers coated PET (lower picture) 